\title{
Thickness Estimation of Discrete Tree-Like Tubular Objects: Application to Vessel Quantification
}

\author{
D. Chillet ${ }^{1,2,3}$, N. Passat ${ }^{1,2}$, M.-A. Jacob-Da $\mathrm{Col}^{1}$, and J. Baruthio ${ }^{2}$ \\ 1 LSIIT, UMR 7005 CNRS-ULP, Parc d'Innovation, \\ bd Sébastien Brant BP 10413, 67412 Illkirch cedex, France \\ \{passat, dacol\}@lsiit.u-strasbg.fr \\ 2 IPB, UMR 7004 CNRS-ULP, Faculté de Médecine, \\ 4, Rue Kirschleger, 67085 Strasbourg cedex, France \\ baruthio@ipb.u-strasbg.fr \\ 3 École Supérieure Chimie Physique Électronique de Lyon, \\ Domaine Scientifique de la Doua, 43, bd du 11 novembre 1918, \\ BP 2077, 69616 Villeurbanne cedex, France \\ dini.chillet@cpe.fr
}

\begin{abstract}
Thickness estimation of discrete objects is often a critical step for shape analysis and quantification in medical applications. In this paper, we propose an approach to estimate the thickness (diameter or cross-section area) of discrete tree-like tubular objects in 3D binary images. The estimation is performed by an iterative process involving skeletonization, skeleton simplification, discrete cross-section plane evaluation and finally area estimation. The method is essentially based on discrete geometry concepts (skeleton, discrete planes, and discrete area). It has been validated on phantoms in order to determine its robustness in case of thickness variations along the studied object. The method has also been applied for vessel quantification and computer-aided diagnosis of vascular pathologies in angiographic data, providing promising results.
\end{abstract}

Keywords: thickness estimation, discrete tree-like tubular objects, vascular imaging, vessel quantification, computer-aided diagnosis.

\section{Introduction}

Thickness estimation (diameter or cross-section area) of discrete objects is often an important step for shape analysis and quantification in medical applications. Since vascular diseases are one of the most important public health problems, quantification of discrete tubular structures can be of precious use in computeraided diagnosis. Indeed, it can help detecting aneurysms and stenoses which are characterized by vessel thickness abnormal variations.

Several methods have been proposed in order to compute tubular object thickness for vessel quantification. Some of them are designed to directly process 
grey-level images [6,7] while others are devoted to segmented binary data [3, 8, 12. The method presented in this paper is devoted to thickness estimation of discrete tree-like tubular objects contained in binary images.

It is composed of the following steps. First, a skeleton is extracted from the initial object. This skeleton is then simplified by a maximum-path pruning process and smoothed with Bézier curves in order to obtain the object medial axes. Tangent lines are then estimated along these medial axes in order to determine the object discrete normal planes. Thickness is finally deduced from the area of these cross-section planes.

The method, devoted to vascular structures (but applicable for any tree-like discrete object) has been validated on tubular phantoms in order to determine the relative estimation errors in case of thickness variations along the object. Finally, real angiographic data have been processed to evaluate the ability of the method to be used for quantification and computer-aided diagnosis.

This paper is organized as follows. In Section 2, previous approaches for thickness estimation of discrete tubular objects from binary images are described. The proposed algorithm is then fully described in Section 3. In Section 4, experimental results obtained from phantoms and real data are provided. Discussion and future projects are finally presented in Section 5.

\section{Related Work}

The main researches concerning 3D tubular object thickness estimation have focused on vascular image analysis, in order to quantify vessel attributes. Two different approaches have been proposed. The first one consists in directly working on 3D angiographic images and to extract thickness information from highintensity grey-level structures [6,7]. It can then take advantage of knowledge concerning the acquisition process. Nevertheless, it can not be used for other application fields. The second approach deals with binary images and can then be used for any segmented grey-level images but also for any application involving virtual or real binary data. The principal methods belonging to this category use the following steps: skeleton extraction, skeleton pruning to obtain the medial axis, and final estimation of a thickness parameter (area or diameter).

In [3], Chen et al. obtain the skeleton by using an iterative thinning algorithm. The skeleton is pruned in order to delete cycles and irrelevant branches. However, they do not focus on quantifying the vessels, only explaining that the diameter can be estimated from the cross-section area.

In a method proposed by Sorantin et al. in [12, the skeleton is obtained with the same thinning algorithm. During the pruning step, the shortest path between two points chosen by the user is determined. Then, the tangent lines along the skeleton are smoothed using the Casteljau's algorithm. The cross-section area is then computed along the object.

An original approach is proposed by Nyström et al. in 8 . The segmented vessels are first reduced to a reversible surface skeleton. A curve skeleton is then extracted from the surface skeleton. Finally, the minimal radius at each point of 
the curve skeleton is estimated using the distance map associated to the initial binary image.

Despite their originality, it has to be noticed that these strategies present several drawbacks. In [3], the method provides a whole skeleton of the tubular tree, but thickness estimation is not explicitly provided. In [12, the proposed approach only processes a single vessel among the whole vascular network. Such a method can then be used for analysing a precise vessel but it requires user interaction for choosing extremity points. Finally, the method proposed in [8], being based on distance maps can only determine the minimal diameter of a non circular cross-section, only allowing to detect vessel narrowings (stenoses) but no vessel thickenings (aneurysms) in medical applications.

In the following, we describe an algorithm allowing to fix these problems. The method enables to compute a whole tree-like structure in order to obtain an accurate thickness estimation along all the branches without any user interaction. This method, essentially based on discrete geometry concepts, is described in the following sections.

\section{Algorithm}

\subsection{Input and Output}

Any 3D binary image $I$ of dimensions $\operatorname{dim}_{x}, \operatorname{dim}_{y}, \operatorname{dim}_{z}$, processed by the method can be considered as a subset of the 3D discrete space: $I=\left[0\right.$, dim $_{x}-$ 1] $\times\left[0, \operatorname{dim}_{y}-1\right] \times\left[0, \operatorname{dim}_{z}-1\right] \subset \mathbb{Z}^{3}$. It has to be noticed that the considered images are assumed to be isotropic (since real data acquisition processes generally provide 3D images with non cubic voxels, such images have to be preprocessed). However, no hypothesis has to be done on the voxel size. In the following, we will always consider the (26,6)-adjacency (26-adjacency for the foreground and 6 -adjacency for the background).

The method takes as input binary images (here obtained with region-growing methods described in [9]). As output, it provides two kinds of data. The first one is a $3 \mathrm{D}$ image identical to the input image but presenting for each voxel a color depending on the object thickness at the current position. The second one is a graph indicating the thickness along the object. These two data then provide both qualitative and quantitative results. The different steps of the proposed method are illustrated on a simple shape example in Fig. 1.

\subsection{Skeleton}

Skeletonization is a process modifying a given object into a thinner object, centered in the initial one. The skeleton is composed of much less points but is assumed to preserve the initial object shape and topology. The proposed method uses an iterative thinning algorithm to perform the skeletonization. At each iteration, only simple voxels (voxels whose deletion does not modify the object topology) are considered and deleted. This first step then provides a skeleton representing the initial object. 

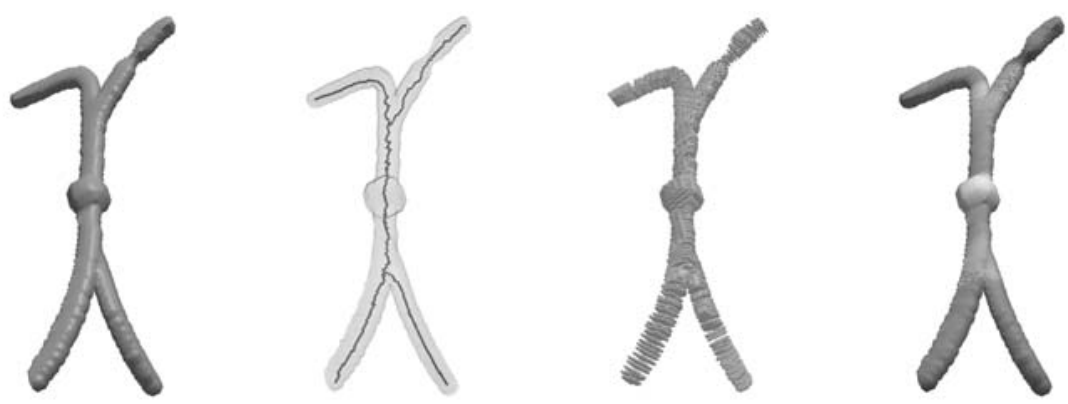

Fig. 1. Successive steps of the method applied on a virtual object. From left to right: initial object; skeleton computation; cross section planes computed by intersecting the object and the digital naive normal planes; thickness image obtained from area estimation (the grey-levels depend on the estimated area of the cross-section planes)

Since skeletonization is a noise-sensitive process, it might happen that the obtained skeleton contains irrelevant branches and non unit-width parts. The algorithm used to remove such branches and useless points is inspired from the method used in [3]. This method is based on an iterative longest path detection algorithm which requires a seed point. In [3], it is proposed to choose the point of highest intensity in the initial image. Nevertheless, since the processed data are binary images, we propose to choose a voxel belonging to the object and located on its bounding box, avoiding an interactive choice. Then, at each iteration, the longest path among all non visited points is searched, using Dijkstra's algorithm, and added to the pruned skeleton. The process is iterated until the longest path becomes shorter than a predetermined threshold.

\subsection{Medial Axes}

Once the skeleton is pruned, the tangent lines have to be computed for each point. The skeleton generally presents an irregular shape. Then, a direct estimation of the tangent lines from it can lead to incorrect results. In order to fix this problem each branch of the skeleton is smoothed by estimating a Bézier curve from it. The control points used for each branch are chosen by sampling the skeleton. The use of Bézier curves enables to significantly smooth the skeleton, finally providing medial axes being correctly oriented and centered in the object. It is then possible to efficiently determine the tangent line in each position of these axes by only considering a set of successive points.

\subsection{Normal Planes}

At this stage of the method, the tangent lines are determined for each point of the medial axes. The tangent at a point defines the normal vector $\boldsymbol{n}=\left(n_{x}, n_{y}, n_{z}\right)$ of the cross-section plane at the current position. We propose to use the digital naive plane definition introduced by Reveillès in [11] to construct the cross- 
section plane for each point. Using digital naive planes presents several advantages. Indeed, fast and efficient algorithms can be used to compute the number of voxels composing them. Moreover, they can be projected on orthogonal planes without loss of information. The cross-section planes are then obtained by intersecting the object and the computed naive planes.

\subsection{Cross-Section Area}

Every cross-section plane can be directly projected onto the three principal planes $O_{y z}, O_{x z}$, and $O_{x y}$. The corresponding areas $A_{x}, A_{y}$, and $A_{z}$ are then estimated using a method proposed in [5. For each projection, the area is estimated as a weighted sum of the pixels (projections of voxels), the weights depending on the pixel neighborhood configurations. Finally, the area of the cross-section plane $A$ is computed as a combination of the three projected areas $A_{x}, A_{y}, A_{z}$, and the coordinates of the normal vector of the cross section plane $\boldsymbol{n}=\left(n_{x}, n_{y}, n_{z}\right)$ :

$$
A=A_{x} \cdot n_{x}+A_{y} \cdot n_{y}+A_{z} \cdot n_{z},
$$

using a formula proposed by Coeurjolly in 4 . For cross-section planes assumed to present a circular or nearly circular shape, the diameter can be directly obtained from the area value.

\section{Experiments and Results}

The proposed method has been implemented on the Medimax 1$]$ software platform and use the ImLib3 $\mathrm{D}^{2}$ open source $\mathrm{C}++$ library. The computer used to run the method was composed of a $3 \mathrm{GHz}$ Pentium IV processor with 2 GB of memory. The algorithm then requires from 10 seconds for simple objects (Fig. 1) to 6 minutes for complex real objects (left picture of Fig. 2).

\subsection{Error Estimation on Phantoms}

The phantoms used for thickness error estimation are cylinders of length varying from 6 to $8 \mathrm{~cm}$, simulating stenoses (narrowings) and aneurysms (thickenings) or having homogeneous diameters. They were created with silicon elastomer according to the method described by Stevanov et al. in [13. The cylinder images have been acquired on a 1 Tesla scanner. The estimated thicknesses have then been compared to the thicknesses directly measured on the phantoms.

The results of error estimation are illustrated in Table 1. Since the first three cylinders (tubes 1,2, and 3) do not present diameter variations, the average estimated diameter is directly compared with the real diameter. The highest relative error is $5.6 \%$ which can be considered as a satisfactory result. The other cylinders (tubes 4, 5, and 6) present thickness variations modeling aneurysms (tube 6) and stenoses (tubes 4 and 5). Both smallest and greatest diameters

\footnotetext{
${ }^{1}$ Available at http://www-ipb.u-strasbg.fr.

2 Available at http://imlib3d.sourceforge.net.
} 

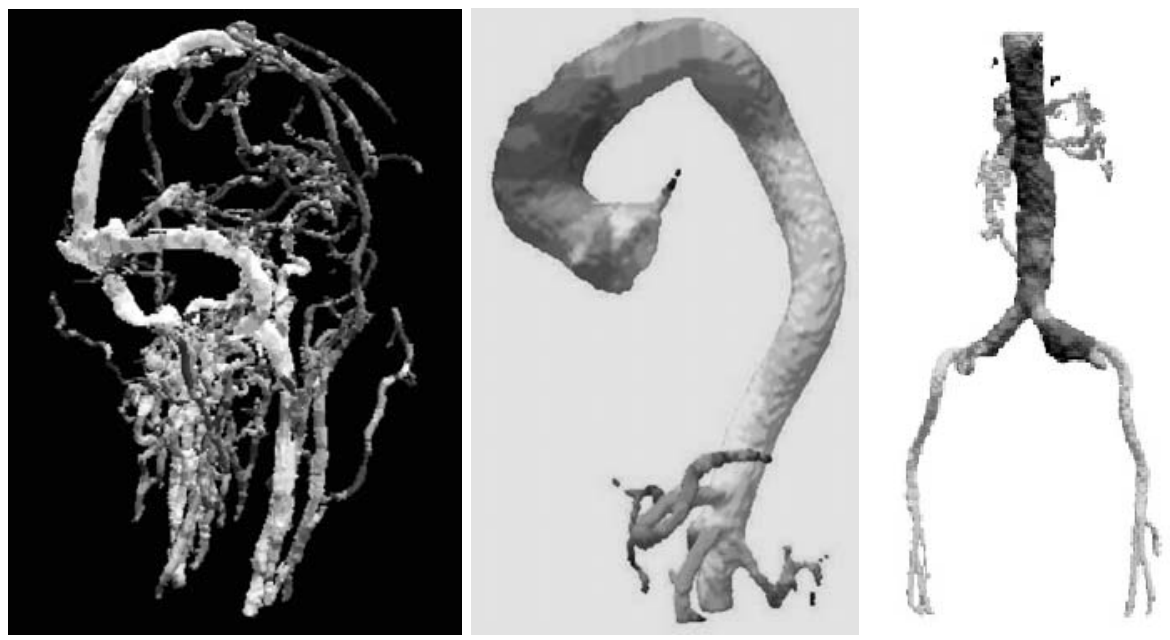

Fig. 2. Vessel quantification of angiographic data. From left to right: cerebral veins and arteries, arch of aorta, iliac bifurcation

Table 1. Phantom thickness estimation errors: real and estimated diameters of phantoms

\begin{tabular}{|c|c|c|c|c|c|}
\hline & \multicolumn{2}{|c|}{ estimated diameter } & \multirow{2}{*}{\multicolumn{2}{|c|}{$\begin{array}{r}\text { real diameter } \\
(\mathrm{mm})\end{array}$}} & \multirow{2}{*}{$\begin{array}{l}\text { error } \\
(\%)\end{array}$} \\
\hline & (voxels) & $(\mathrm{mm})$ & & & \\
\hline & 34.12 & 30 . & & 29.00 & \\
\hline & 10.64 & & & 9.80 & \\
\hline & 5.4 & 4.93 & & 5.00 & \\
\hline & $6.13 / 9.81$ & $5.51 / 8.81$ & 5.70 & 9.80 & $0.6 / 10$ \\
\hline & $16.54 / 23.00$ & $14.85 / 20.65$ & 15.00 & 20.50 & $1.0 / 0$ \\
\hline & $16.37 / 22.84$ & $14.65 / 20.51$ & 14.50 & 20.20 & $1.3 / 1$ \\
\hline
\end{tabular}

Table 2. Phantom thickness estimation errors: real and estimated degrees of severity of simulated stenoses and aneurysms

\begin{tabular}{|l|r|r|r|}
\cline { 2 - 4 } \multicolumn{1}{l|}{} & \multicolumn{2}{|c|}{ degree of severity } & error \\
\cline { 2 - 4 } \multicolumn{1}{l|}{ estimated } & real & $(\%)$ \\
tube 4 & 0.37 & 0.42 & 11.9 \\
tube 5 & 0.28 & 0.27 & 3.7 \\
tube 6 & 0.28 & 0.28 & 0.0 \\
\hline
\end{tabular}

are compared in Table 1, while the real and estimated degrees of severity (an important criterion for diagnosis) are detailed in Table 2. This degree is defined by $1-\frac{\min }{\max }$ where $\min$ (resp. $\max$ ) is the smallest (resp. the largest) diameter. One can observe that most results are quite accurate (errors are generally lower 
than $2 \%$ ). Only one result is uncorrect, for tube 4 (error of approximately $10 \%$ ). However, this error has been caused by a skeletonization error at the extremity of the cylinder which can be explained by the low length/diameter ratio of the phantom. In real data, the tubular structures being much longer, this kind of skeletonization errors do not occur.

\subsection{Vessel Quantification and Computer-Aided Diagnosis}

The method has been tested on real angiographic data (cerebral and carotid MRA, thoracic and abdominal CT scan) for vessel quantification or computeraided diagnosis of vascular pathologies (aneurysms and stenoses). Examples of vessel quantification are illustrated for several vascular structures in Fig. 2,

This figure emphasizes the visualization improvement provided by thickness information, which makes the 3D data more easily readable than binary data. It has to be noticed that information on vessel size and orientation (provided by the estimated medial axes) is also of precious use for cerebral vascular atlas generation methods as the one proposed in [10]. However, quantification can also be used for computer-aided diagnosis, as described in the following example.

In the left picture of Fig. 3, a maximum intensity projection (MIP) of an iliac bifurcation angiography can be observed. For this data, the method has provided an image where the color (here the grey-level) depends on the thickness (right picture of Fig. 2), but also a graph representing the thickness (right part of Fig. 3). On the $3 \mathrm{D}$ colored image, it is possible to visually detect a dark part on the right branch, surrounded by two clear parts: this structure is the sought aneurysm. On the graph, the curves representing the thickness evolution along the two branches are quite similar apart a peak: this difference corresponds with
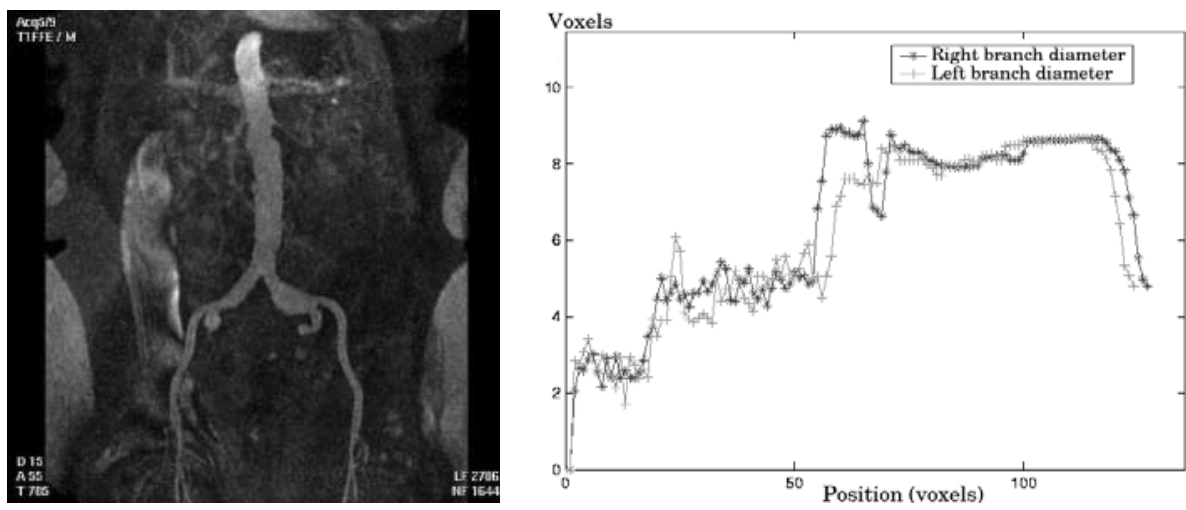

Fig. 3. Iliac bifurcation thickness estimation. Left: MIP showing an aneurysm on the right branch (see right picture of Figure 2 for the corresponding 3D visualization). Right: the right and left branches diameter evolution according to the position 
the localisation of the aneurysm. The graph enables to determine not only its lenght but also its severity degree.

\section{$5 \quad$ Discussion and Further Works}

This paper has proposed a fully automatic method for thickness estimation of discrete tree-like tubular structures in binary images. This method, devoted to $3 \mathrm{D}$ vessel analysis and quantification, can however be used for any kind of objects presenting similar properties. The algorithm, essentially based on discrete geometry concepts presents several advantages by comparison to previous approaches: automation, ability to process a whole tree-like object and accurate estimation of cross-section areas. This accuracy has been validated on phantoms, emphasizing the method robustness even in case of thickness variations along the object. Tests have also been carried out on real angiographic images. They tend to prove that the method can be useful for vessel quantification and computer-aided diagnosis of vascular pathologies such as aneurysms and stenoses. Further work could now consist in modifying and improving the skeleton smoothing step, currently using Bézier curves, in order to obtain a method entirely based on discrete structures.

\section{Acknowledgement}

The authors thank the EPML IRMC 3 (Équipe Projet Multi-Laboratoires Imagerie et Robotique Médicale et Chirurgicale, EPML \#9 CNRS-STIC) for its financial support.

\section{References}

1. G. Borgefors, I. Nyström, G. Sanniti di Baja: Computing skeletons in three dimensions. Pattern Recognition 32 (1999) 1225-1236

2. S.Y.J. Chen, J.D. Caroll, J.C. Messenger: Quantitative analysis of reconstructed $3 \mathrm{D}$ coronary arterial tree and intracoronary devices. IEEE Transactions on Medical Imaging 21 (2002) 724-740

3. Z. Chen, S. Molloi: Automatic 3D vascular tree construction in CT angiography. Computerized Medical Imaging and Graphics 27 (2003) 469-479

4. D. Coeurjolly: Algorithmique et géometrie discrète pour la caractérisation des courbes et des surfaces. PhD Thesis, Université Lumière, Lyon 2 (2002). http://liris.cnrs.fr/david.coeurjolly/These/these.pdf

5. O. Duda, P.E. Hart: Pattern Classification and Scene Analysis. John Wiley \& Sons Inc. (1973)

6. A.F. Frangi, W.J. Niessen, P.J. Nederkoorn, J. Bakker, W.P.T.M. Mali, M.A. Viergever. Quantitative analysis of vascular morphology from 3D MR angiograms: in vitro and in vivo results. Magnetic Resonance in Medicine 45 (2001) $311-322$

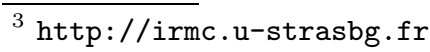


7. R.M. Hoogeveen, C.J.G. Bakker, W. Mali, M.A. Viergever: Vessel diameter measurement in TOF and PC angiography: a need for standardization. In Annual Meeting of the International Society for Magnetic Resonance in Medicine (1997) 1847

8. I. Nyström, O. Smedby: New presentation method for magnetic resonance angiography images based on skeletonization. In Proc. SPIE Medical Imaging: Image Display and Visualization 20003976 (2000) 515-522

9. N. Passat, C. Ronse, J. Baruthio, J.-P. Armspach, C. Maillot, C. Jahn: Atlas-based method for segmentation of cerebral vascular trees from phase-contrast magnetic resonance angiography. In Proc. SPIE Medical Imaging: Image Processing 2004 5370 (2004) 420-431

10. N. Passat, C. Ronse, J. Baruthio, J.-P. Armspach, C. Maillot: Cerebral vascular atlas generation for anatomical knowledge modeling and segmentation purpose. To appear in IEEE Computer Vision and Pattern Recognition (2005)

11. J.P. Reveillès: Combinatorial pieces in digital lines and planes. In Proc. Vision Geometry IV (1995) 23-34

12. E. Sorantin, C. Halmai, B. Erdohelyi, K. Palagyi, L.G. Nyul, K. Olle, B. Geiger, F. Lindbichler, G. Friedrich, K. Kiesler: Spiral-CT-based assessment of tracheal stenoses using 3D skeletonization. IEEE Transactions on Medical Imaging 21 (2002) 263-273

13. M. Stevanov, J. Baruthio, B. Eclancher: Fabrication of elastomer arterial models with specified compliance. Journal of Applied Physiology 88 (2000) 1291-1294 